\title{
CORRIGENDUM
}

\section{AVERAGING FORMULA FOR NIELSEN NUMBERS OF MAPS ON INFRA-SOLVMANIFOLDS OF TYPE (R) - CORRIGENDUM}

\author{
KU YONG HA AND JONG BUM LEE
}

First published online by Duke University Press 15 January 2010, subsequently published online by Cambridge University Press 11 January 2016, doi:10.1017/S0027763000009818

The authors gave an example showing an error in [2, Lemma 3.3], and below offer at least a partial correction for that error under the unimodularity assumption. This makes all of the remaining results in [2] valid.

Consider the three-dimensional solvable non-unimodular Lie algebra $\mathfrak{S}$ :

$$
\mathfrak{S}=\mathbb{R}^{2} \rtimes_{\sigma} \mathbb{R}, \quad \text { where } \sigma(t)=\left[\begin{array}{ll}
t & 0 \\
0 & t
\end{array}\right] .
$$

This Lie algebra has a faithful matrix representation as follows:

$$
\left[\begin{array}{llll}
s & 0 & 0 & a \\
0 & s & 0 & b \\
0 & 0 & 0 & s \\
0 & 0 & 0 & 0
\end{array}\right]
$$

We can choose an ordered (linear) basis for $\mathfrak{S}$ :

$$
\mathbf{b}_{1}=\left[\begin{array}{cccc}
0 & 0 & 0 & 1 \\
0 & 0 & 0 & 0 \\
0 & 0 & 0 & 0 \\
0 & 0 & 0 & 0
\end{array}\right], \quad \mathbf{b}_{2}=\left[\begin{array}{cccc}
0 & 0 & 0 & 0 \\
0 & 0 & 0 & 1 \\
0 & 0 & 0 & 0 \\
0 & 0 & 0 & 0
\end{array}\right], \quad \mathbf{b}_{3}=\left[\begin{array}{cccc}
1 & 0 & 0 & 0 \\
0 & 1 & 0 & 0 \\
0 & 0 & 0 & 1 \\
0 & 0 & 0 & 0
\end{array}\right]
$$

2010 Mathematics subject classification. 55M20, 57S30.

The second-named author is supported in part by Basic Science Researcher Program through the National Research Foundation of Korea (NRF) funded by the Ministry of Education (No. 2013R1A1A2058693).

(C) 2016 by The Editorial Board of the Nagoya Mathematical Journal 
They satisfy $\left[\mathbf{b}_{1}, \mathbf{b}_{2}\right]=\mathbf{0},\left[\mathbf{b}_{3}, \mathbf{b}_{1}\right]=\mathbf{b}_{1}$ and $\left[\mathbf{b}_{3}, \mathbf{b}_{2}\right]=\mathbf{b}_{2}$. The connected and simply connected solvable Lie group $S$ associated with the Lie algebra $\mathfrak{S}$ is

$$
S=\left\{\left[\begin{array}{cccc}
e^{t} & 0 & 0 & x \\
0 & e^{t} & 0 & y \\
0 & 0 & 1 & t \\
0 & 0 & 0 & 1
\end{array}\right] \mid x, y, t \in \mathbb{R}\right\} .
$$

Let $g=((x, y), t)$ denote an element of $S$. Because $\operatorname{Ad}(g): \mathfrak{S} \rightarrow \mathfrak{S}$ is given by $\operatorname{Ad}(g)(A)=g A g^{-1}$ for $A \in \mathfrak{S}$, a simple computation shows that the adjoint of $g$ is given by

$$
\operatorname{Ad}(g)=\left[\begin{array}{ccc}
e^{t} & 0 & -x \\
0 & e^{t} & -y \\
0 & 0 & 1
\end{array}\right] .
$$

Let $\varphi$ be a Lie algebra homomorphism on $\mathfrak{S}$. Since $[\mathfrak{S}, \mathfrak{S}]$ is generated by $\mathbf{e}_{1}$ and $\mathbf{e}_{2}$, we have

$$
\begin{aligned}
& \varphi\left(\mathbf{b}_{1}\right)=m_{11} \mathbf{b}_{1}+m_{21} \mathbf{b}_{2}, \\
& \varphi\left(\mathbf{b}_{2}\right)=m_{12} \mathbf{b}_{1}+m_{22} \mathbf{b}_{2}, \\
& \varphi\left(\mathbf{b}_{3}\right)=p \mathbf{b}_{1}+q \mathbf{b}_{2}+m \mathbf{b}_{3}
\end{aligned}
$$

for some $m_{i j}, p, q, m \in \mathbb{R}$. Since $\varphi$ preserves the bracket operations $\left[\mathbf{b}_{3}, \mathbf{b}_{1}\right]=$ $\mathbf{b}_{1}$ and $\left[\mathbf{b}_{3}, \mathbf{b}_{2}\right]=\mathbf{b}_{2}$, it follows easily that

$$
\begin{array}{ll}
m_{11}(m-1)=0, & m_{12}(m-1)=0, \\
m_{21}(m-1)=0, & m_{22}(m-1)=0 .
\end{array}
$$

Therefore, with respect to the basis $\left\{\mathbf{b}_{1}, \mathbf{b}_{2}, \mathbf{b}_{3}\right\}$ of $\mathfrak{S}, \varphi$ is one of the following:

$$
\begin{aligned}
\text { Type (I) } & {\left[\begin{array}{ccc}
m_{11} & m_{12} & p \\
m_{21} & m_{22} & q \\
0 & 0 & 1
\end{array}\right] } \\
\text { Type (II) } & {\left[\begin{array}{ccc}
0 & 0 & p \\
0 & 0 & q \\
0 & 0 & m
\end{array}\right] \quad \text { with } m \neq 1 . }
\end{aligned}
$$

Now we can easily check that

$$
\operatorname{det}(\varphi-I)= \begin{cases}0 & \text { when } \varphi \text { is of type (I), } \\ m-1 & \text { when } \varphi \text { is of type (II) }\end{cases}
$$




$$
\operatorname{det}(\varphi-\operatorname{Ad}(g))= \begin{cases}0 & \text { when } \varphi \text { is of type (I) } \\ e^{2 t}(m-1) & \text { when } \varphi \text { is of type (II) }\end{cases}
$$

This example shows that [2, Lemma 3.3] is not true in general. We remark also that $S$ is not unimodular, and hence, as can be expected, $\operatorname{det}(\operatorname{Ad}(g))=$ $e^{2 t} \neq 1$ for all $t \neq 0$. We prove, however, that the lemma is true under the unimodularity assumption of the connected Lie group. That is, the following theorem.

THEOREM 1. Let $S$ be a connected and simply connected solvable Lie group, and let $D: S \rightarrow S$ be a Lie group homomorphism. If $S$ is unimodular, then for any $x \in S$,

$$
\operatorname{det}\left(I-D_{*}\right)=\operatorname{det}\left(I-\operatorname{Ad}(x) D_{*}\right) .
$$

REMARK 2. It is known that if a Lie group admits a lattice (discrete cocompact subgroup), then it is unimodular. Consequently, the remaining results of [2] are valid.

LEMMA 3. Let $S$ be a connected and simply connected solvable Lie group, and let $D: S \rightarrow S$ be a Lie group homomorphism. Then, for any $x \in S$, $I-D_{*}$ is an isomorphism if and only if $I-\operatorname{Ad}(x) D_{*}$ is an isomorphism.

Proof. Because $I-\operatorname{Ad}\left(x^{-1}\right) \operatorname{Ad}(x) D_{*}=I-D_{*}$, it suffices to show the only if.

Let $G=[S, S]$; then $G$ is nilpotent, and $S / G \cong \mathbb{R}^{k}$ for some $k$. Then we have the following commutative diagram:

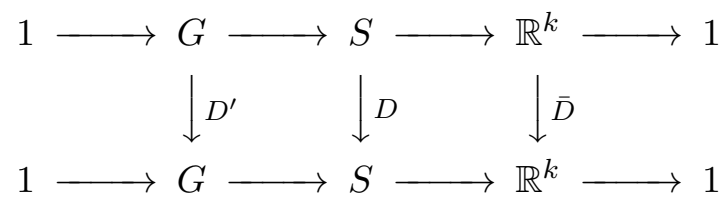

This induces the following commutative diagram:

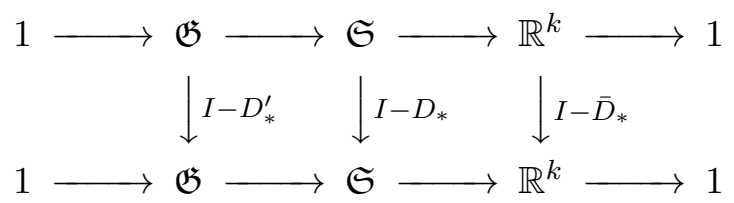

For $x \in S$, we denote by $\tau_{x}$ the inner automorphism on $S$ whose differential is $\operatorname{Ad}(x)$. This induces an automorphism on $G$, and we still 
denote it by $\tau_{x}$ and its differential is $\operatorname{Ad}^{\prime}(x)$. Then we can express $I-D_{*}$ and $I-\operatorname{Ad}(x) D_{*}$ as

$$
\begin{gathered}
I-D_{*}=\left[\begin{array}{cc}
I-\bar{D}_{*} & 0 \\
* & I-D_{*}^{\prime}
\end{array}\right], \\
I-\operatorname{Ad}(x) D_{*}=\left[\begin{array}{cc}
I-\bar{D}_{*} & 0 \\
* & I-\operatorname{Ad}^{\prime}(x) D_{*}^{\prime}
\end{array}\right]
\end{gathered}
$$

with respect to some linear basis for $\mathfrak{S}$.

Assume that $I-\bar{D}_{*}$ is an isomorphism. We claim that $I-D_{*}^{\prime}$ is an isomorphism if and only if $I-\operatorname{Ad}^{\prime}(x) D_{*}^{\prime}$ is an isomorphism.

Since $I-\bar{D}$ is an isomorphism on $\mathbb{R}^{k}, \operatorname{fix}(\bar{D})=\operatorname{ker}(I-\bar{D})$ is a trivial group. For any $x \in S$, we consider the exact sequence of the Reidemeister sets

$$
\mathcal{R}\left[\tau_{x} D^{\prime}\right] \stackrel{\hat{i}^{x}}{\longrightarrow} \mathcal{R}\left[\tau_{x} D\right] \stackrel{\hat{p}^{x}}{\longrightarrow} \mathcal{R}[\bar{D}] \longrightarrow 1 ;
$$

$\hat{p}^{x}$ is surjective, and $\left(\hat{p}^{x}\right)^{-1}([\overline{1}])=\operatorname{im}\left(\hat{i}^{x}\right)$. If $\hat{i}^{x}\left(\left[g_{1}\right]\right)=\hat{i}^{x}\left(\left[g_{2}\right]\right)$ for some $g_{1}, g_{2} \in G$, then by definition there is $y \in S$ such that $g_{2}=y g_{1}\left(\tau_{x} D(y)\right)^{-1}$. The image in $S / G$ is then $\bar{g}_{2}=\bar{y} \bar{g}_{1} \bar{D}(\bar{y})^{-1}$, which yields that $\bar{y} \in \operatorname{fix}(\bar{D})=$ $\{\overline{1}\}$, and so $y \in G$. This shows that $\hat{i}^{x}$ is injective for all $x \in S$. Because there is a bijection between the Reidemeister sets $\mathcal{R}[D]$ and $\mathcal{R}\left[\tau_{x} D\right]$ given by $[g] \mapsto\left[g x^{-1}\right]$, it follows that $R\left(D^{\prime}\right)=R\left(\tau_{x} D^{\prime}\right)$. On the other hand, by $[1$, Lemma 3.4], since $I-\bar{D}_{*}$ is an isomorphism, $R(\bar{D})<\infty$, and

$$
\begin{aligned}
I-\operatorname{Ad}^{\prime}(x) D_{*}^{\prime} \text { is an isomorphism } & \Longleftrightarrow R\left(\tau_{x} D^{\prime}\right)<\infty, \\
I-D_{*}^{\prime} \text { is an isomorphism } & \Longleftrightarrow R\left(D^{\prime}\right)<\infty .
\end{aligned}
$$

This proves our claim.

Now assume that $I-D_{*}$ is an isomorphism. Then it follows that $I-\bar{D}_{*}$ and $I-D_{*}^{\prime}$ are isomorphisms. By the above claim, $I-\operatorname{Ad}^{\prime}(x) D_{*}^{\prime}$, and hence $I-\operatorname{Ad}(x) D_{*}$ are isomorphisms.

Proof of Theorem 1. If $S$ is Abelian, then $\operatorname{Ad}(x)$ is the identity and hence there is nothing to prove. We may assume that $S$ is non-Abelian. Further, by Lemma 3, we may assume that $I-D_{*}$ is an isomorphism. Hence, $I-\bar{D}_{*}$ and $I-\operatorname{Ad}(x) D_{*}$ are isomorphisms for all $x \in S$.

Denote $G=[S, S]$ and $\Lambda_{0}=S / G$. Then $G$ is nilpotent, and $\Lambda_{0} \cong \mathbb{R}^{k_{0}}$ for some $k_{0}>0$. Consider the lower central series of $G$ :

$$
G=\delta_{1}(G) \supset \delta_{2}(G) \supset \cdots \supset \delta_{c}(G) \supset \delta_{c+1}(G)=1,
$$


where $\delta_{i+1}(G)=\left[G, \delta_{i}(G)\right]$. Let $\Lambda_{i}=\delta_{i}(G) / \delta_{i+1}(G)$. Then $\Lambda_{i} \cong \mathbb{R}^{k_{i}}$ for some $k_{i}>0$. For each $x \in S$, the conjugation $\tau_{x}$ by $x$ induces an automorphism on $G$. Since each $\delta_{i}(G)$ is a characteristic subgroup of $G, \tau_{x} \in \operatorname{Aut}(G)$ restricts to an automorphism on $\delta_{i}(G)$, and hence on $\Lambda_{i}$. Now, if $x \in G$, then we have observed that the induced action on $\Lambda_{i}$ is trivial. Consequently, there is a well-defined action of $\Lambda_{0}=S / G$ on $\Lambda_{i}$. Hence, there is a well-defined action of $\Lambda_{0}$ on $\Lambda_{i}$. This action can be viewed as a homomorphism $\mu_{i}: \Lambda_{0} \rightarrow$ $\operatorname{Aut}\left(\Lambda_{i}\right)$. Note that $\mu_{0}$ is trivial. Moreover, for any $x \in S$ denoting its image under $S \rightarrow \Lambda_{0}$ by $\bar{x}$, the differential of conjugation $\tau_{x}$ by $x$ can be expressed as a matrix of the form

$$
\operatorname{Ad}(x)\left(=\tau_{x *}\right)=\left[\begin{array}{cccc}
I & 0 & \cdots & 0 \\
* & \mu_{1}(\bar{x}) & \cdots & 0 \\
\vdots & \vdots & \ddots & \vdots \\
* & * & \cdots & \mu_{c}(\bar{x})
\end{array}\right]
$$

by choosing a suitable basis of the Lie algebra $\mathfrak{S}$ of $S$.

The homomorphism $D: S \rightarrow S$ induces homomorphisms $D_{i}: \delta_{i}(G) \rightarrow$ $\delta_{i}(G)$ and hence homomorphisms $\bar{D}_{i}: \Lambda_{i} \rightarrow \Lambda_{i}$, so that the following diagram is commutative:

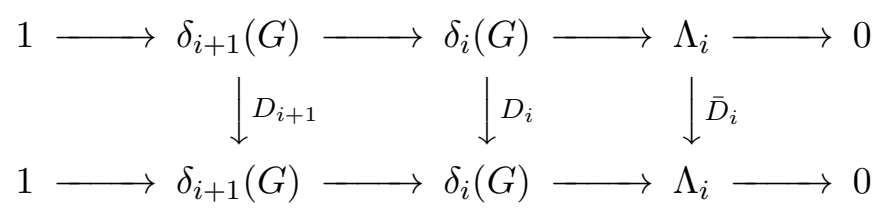

Hence, the differential of $D$ can be expressed as a matrix of the form

$$
D_{*}=\left[\begin{array}{cccc}
\bar{D}_{0} & 0 & \cdots & 0 \\
* & \bar{D}_{1} & \cdots & 0 \\
\vdots & \vdots & \ddots & \vdots \\
* & * & \cdots & \bar{D}_{c}
\end{array}\right]
$$

with respect to the same basis for $\mathfrak{S}$ chosen as above.

Furthermore, the above commutative diagram produces the following identities:

$$
\bar{D}_{i} \circ \mu_{i}(\bar{x})=\mu_{i}\left(\bar{D}_{0}(\bar{x})\right) \circ \bar{D}_{i}, \quad \forall \bar{x} \in \Lambda_{0}, \forall i=0,1, \ldots, c .
$$

Let $x \in S$ with $\bar{x} \in \Lambda_{0}=\mathbb{R}^{k_{0}}$. Since $I-\bar{D}_{0}: \mathbb{R}^{k_{0}} \rightarrow \mathbb{R}^{k_{0}}$ is invertible, we can choose $\bar{y} \in \Lambda_{0}$ so that $\left(I-\bar{D}_{0}\right)(\bar{y})=\bar{x}$. Now, using the above identities, 
we observe that

$$
\begin{aligned}
\operatorname{det}\left(I-\mu_{i}(\bar{x}) \bar{D}_{i}\right) & =\operatorname{det}\left(\mu_{i}(\bar{y}) \mu_{i}(\bar{y})^{-1}-\mu_{i}(\bar{x}) \mu_{i}\left(\bar{D}_{0}(\bar{y})\right) \bar{D}_{i} \mu_{i}(\bar{y})^{-1}\right) \\
& =\operatorname{det}\left(\mu_{i}(\bar{y}) \mu_{i}(\bar{y})^{-1}-\mu_{i}\left(\bar{x}+\bar{D}_{0}(\bar{y})\right) \bar{D}_{i} \mu_{i}(\bar{y})^{-1}\right) \\
& =\operatorname{det}\left(\mu_{i}(\bar{y}) \mu_{i}(\bar{y})^{-1}-\mu_{i}(\bar{y}) \bar{D}_{i} \mu_{i}(\bar{y})^{-1}\right) \\
& =\operatorname{det}\left(\mu_{i}(\bar{y})\right) \operatorname{det}\left(I-\bar{D}_{i}\right) \operatorname{det}\left(\mu_{i}(\bar{y})\right)^{-1} \\
& =\operatorname{det}\left(I-\bar{D}_{i}\right) .
\end{aligned}
$$

Consequently, we have

$$
\begin{aligned}
\operatorname{det}\left(I-\operatorname{Ad}(x) D_{*}\right) & =\operatorname{det}\left(I-\bar{D}_{0}\right) \prod_{i=1}^{c} \operatorname{det}\left(I-\mu_{i}(\bar{x}) \bar{D}_{i}\right) \\
& =\operatorname{det}\left(I-\bar{D}_{0}\right) \prod_{i=1}^{c} \operatorname{det}\left(I-\bar{D}_{i}\right)=\operatorname{det}\left(I-D_{*}\right) .
\end{aligned}
$$

This completes the proof of our theorem.

\section{REFERENCES}

[1] K. Dekimpe and P. Penninckx, The finiteness of the Reidemeister number of morphisms between almost-crystallographic groups, J. Fixed Point Theory Appl. 9 (2011), $257-283$.

[2] J. B. Lee and K. B. Lee, Averaging formula for Nielsen numbers of maps on infrasolvmanifolds of type (R), Nagoya Math. J. 196 (2009), 117-134; doi:10.1017/S00277 63000009818.

Ku Yong Ha

Department of Mathematics

Sogang University

Seoul 04107

Korea

kyha@sogang.ac.kr

Jong Bum Lee

Department of Mathematics

Sogang University

Seoul 04107

Korea

jlee@sogang.ac. kr 